\title{
Semi-analytical model for a static sheath including a weakly collisional presheath
}

\section{Tatsuru Shirafuji, Kazuki Denpoh}

\begin{tabular}{|c|l|}
\hline Citation & Japanese Journal of Applied Physics, 57(6S2): 06JG02 \\
\hline Issue Date & 2018-05-08 \\
\hline Type & Journal Article \\
\hline Textversion & author \\
\hline \multirow{3}{*}{ Rights } & $\begin{array}{l}\text { C } 2018 \text { The Japan Society of Applied Physics. } \\
\text { The final publication is available at https://doi.org/10.7567/JJAP.57.06JG02 . } \\
\text { This is the accepted manuscript version. Please cite only the published version. } \\
\text { この論文は出版社版でありません。引用の際には出版社版をご確認ご利用ください。 }\end{array}$ \\
\hline DOI & 10.7567/JJAP.57.06JG02 \\
\hline
\end{tabular}

\author{
Self-Archiving by Author(s) \\ Placed on: Osaka City University
}

Tatsuru Shirafuji and Kazuki Denpoh (2018). Semi-analytical model for a static sheath including a weakly collisional presheath. Japanese Journal of Applied Physics. 57, 06JG02.

https://doi.org/10.7567/JJAP.57.06JG02. 


\title{
Semi-analytical model for a static sheath including a weakly collisional presheath
}

\author{
Tatsuru Shirafuji*1 and Kazuki Denpoh ${ }^{2}$ \\ ${ }^{1}$ Department of Physical Electronics and Informatics, Osaka City University, Osaka 558-8585, Japan \\ 2 Process Development Center, Tokyo Electron Limited, Nirasaki, Yamanashi 407-0192, Japan
}

\begin{abstract}
A semi-analytical static sheath (SASS) model can provide a spatial potential profile on a biased surface with microstructures, which can be used for predicting ion trajectories on the surface. However, two- or three-dimensional SASS models require a search procedure for a sheath edge equipotential profile, at which ions have the Bohm velocity, as the starting positions for calculating ion trajectories. This procedure can be troublesome when surface microstructures have complex structures. This difficulty is due to the fact that the SASS model cannot handle a presheath region. In this work, we propose a modified SASS model that can handle a presheath region. By using the modified SASS model, ion trajectories can be calculated from edges with arbitrary geometry without searching for the equipotential profile corresponding to sheath edges.
\end{abstract}

\section{Introduction}

In reactive ion etching processes, ions are accelerated in a sheath adjacent to a wafer surface and play an important role in controlling etching characteristics such as etching rate, ${ }^{1)}$ anisotropy, ${ }^{2,3)}$ directionality, ${ }^{4-6)}$ selectivity, ${ }^{7,8)}$ and damage. ${ }^{9-12)}$ Among them, the directionality of ions is closely related to the shapes of microstructures formed on the wafer surface. The resulting microstructures can have desirable or undesirable shapes depending on the purpose of the etching process.

In the processes for fabricating ultralarge-scale integrated circuits, perpendicular ion bombardment is generally required. Obliquely impinging ions, which tend to exist near the edge of a wafer, are not preferred because they cause anomalous microstructure formation such as microtrenching ${ }^{13)}$ and shadowing. ${ }^{14)}$ Recent advanced etchers therefore employ an appropriately designed focus ring that surrounds a wafer to compensate for the nonuniformity of impinging ions. ${ }^{15,16)}$ Some other etching processes, on the other hand, prefer oblique ion bombardment, as used in the processes for fabricating three-dimensional (3D) photonic crys-

*E-mail: sirafuji@elec.eng.osaka-cu.ac.jp 
tals. ${ }^{17,18)}$ Regardless of the process, simulation tools are required for analyzing or designing electrical potential profiles governing ion trajectories in a sheath for a given surface geometry.

At present, two types of simulation tools are available. One is a large-scale model covering a whole plasma reactor, in which fluid or particle dynamics are calculated together with reaction kinetics and electromagnetics. ${ }^{15,16,19)}$ Although the large-scale model provides detailed information in a plasma reactor, it is time-consuming and requires days to weeks to accomplish the calculation.

Another is a small-scale model or a stand-alone model, which covers only a sheath region and is based on the classical sheath theory, which was first proposed by Tonks and Langmuir in 1929. ${ }^{20)}$ The stand-alone model was simplified by Bohm in 1949, who deduced Bohm's criterion. ${ }^{21)}$ The stand-alone sheath theory has been critically discussed by many researchers, ${ }^{22-36)}$ even recently. ${ }^{37-46)}$

Although we must pay attention to the problem of the singularity involved in the classical sheath theory, the stand-alone model has an advantage of low calculation cost. In the case of one-dimensional (1D) geometries, we can analytically calculate potential profiles in a sheath adjacent to a wafer surface using the four basic plasma parameters of ion mass $M_{\mathrm{i}}$, bulk plasma density $n_{0}$, electron temperature $T_{\mathrm{e}}$, and bias voltage $V_{\mathrm{dc}}$ on the wafer surface. The stand-alone model is also valid in the case of two-dimensional (2D) or three-dimensional (3D) geometries, although the solutions in these cases are not obtained analytically but numerically. We call the stand-alone model employing numerical calculations a semi-analytical static sheath (SASS) model.

In a SASS model, we assume a calculation area that is sufficiently larger than a roughly estimated sheath and self-consistently solve a Poisson's equation together with several constraints to form a sheath potential profile on a wafer surface. Since we can neglect ion-neutral collisions in the sheath area in most cases, we can calculate ion trajectories by solving the equation of motion for ions in the sheath using the calculated potential profile. Because of this benefit, the 2D or 3D SASS models have been used for various purposes such as explanation of the ion-focusing effect by the sheath, ${ }^{47-49)}$ analysis of the sheath potential in radio-frequency capacitively coupled plasmas, ${ }^{50-52)}$ and explanation of the oblique etching used for fabricating 3D photonic crystals. ${ }^{17,18)}$

However, SASS models have a limitation because the presheath region, which is located between the sheath edge and the bulk plasma, in a solution of a SASS model shows physically unacceptable characteristics as described later. This means that the equation of motion for ions cannot be applied in the entire calculation area and only within the sheath region. Thus, 
when we calculate ion trajectories, we have to search for the sheath edge positions, which are the initial positions of ions with the Bohm velocity, in the entire calculation area. The sheath edge positions form an equipotential line or surface in the case of $2 \mathrm{D}$ or $3 \mathrm{D}$ geometries. It is very easy to find the sheath edge positions in the case of simple geometries such as a plane surface. However, it becomes a time-consuming and troublesome procedure if complex 2D or $3 \mathrm{D}$ objects exist in the calculation area. If we can appropriately treat the presheath region, we can use given geometry edges as the initial positions of ions to calculate their trajectories without troublesome searching procedures for sheath edges. In this paper, we report that a SASS model can treat a presheath region by the appropriate handling of its quasi-neutrality and by assuming weakly collisional conditions.

\section{Theory}

\subsection{Basic equations for conventional SASS}

In the conventional SASS model (model 1 hereafter), we solve the following Poisson's equation:

$$
\nabla^{2} V=-\frac{q_{0}}{\varepsilon_{0}}\left(n_{\mathrm{i}}-n_{\mathrm{e}}\right)
$$

under the following constraints: ${ }^{17,47-52)}$

$$
\begin{aligned}
& n_{\mathrm{e}}= \begin{cases}n_{\mathrm{s}} \exp \left(\frac{q_{0} V}{k_{\mathrm{B}} T_{\mathrm{e}}}\right) & (V \leq 0), \\
n_{\mathrm{s}} & (V>0),\end{cases} \\
& u_{\mathrm{i}}^{2}=u_{\mathrm{B}}^{2}-\frac{2 q_{0} V}{M_{\mathrm{i}}}, \\
& n_{\mathrm{i}}= \begin{cases}n_{\mathrm{s}} \frac{u_{\mathrm{B}}}{u_{\mathrm{i}}} & \left(u_{\mathrm{i}}^{2} \geq u_{\mathrm{B}}^{2}\right), \\
n_{\mathrm{s}} & \left(u_{\mathrm{i}}^{2}<u_{\mathrm{B}}^{2}\right) .\end{cases}
\end{aligned}
$$

Although these equations can be applied to $1 \mathrm{D}, 2 \mathrm{D}$, and 3D geometries, we explain the SASS theory in 1D geometry for simplicity. In these equations, $x$, which is implicitly involved in the differential operator $\nabla$, is the distance from the negatively biased surface. $V$ is the potential measured from the sheath edge. $q_{0}$ is the unit charge. $\varepsilon_{0}$ is the vacuum permittivity. $k_{\mathrm{B}}$ is the Boltzmann constant. $M_{\mathrm{i}}$ is the ion mass. $T_{\mathrm{e}}$ is the electron temperature. $n_{\mathrm{e}}$ is the electron density. $n_{\mathrm{s}}=\mathrm{e}^{-1 / 2} n_{0} \approx 0.6 n_{0}$ is plasma density at the sheath edge, where $n_{0}$ is the bulk plasma density. $u_{\mathrm{i}}$ is the ion velocity. In the case of $2 \mathrm{D}$ or $3 \mathrm{D}$ geometries, $u_{\mathrm{i}}$ is the norm of the ion velocity vector $\boldsymbol{u}_{\mathrm{i}} \cdot u_{\mathrm{B}}$ is the Bohm velocity given by $\sqrt{k_{\mathrm{B}} T_{\mathrm{e}} / M_{\mathrm{i}}}$. 
Since we assume that a negative bias is applied on the surface at $x=0$, we do not accept solutions with positive $V$. Therefore, when $V$ becomes positive during iterations to solve equations, we set $V=0$ as indicated by Eq. (2).

Although the self-consistent solution of model 1 is obtained in the entire calculation area, only a limited area near the surface will have the characteristics of a sheath that satisfies the first equation of Eq. (2), Eq. (3), and the first equation of Eq. (4). If we set a sufficiently large calculation area, the sheath area is within the calculation area. The outermost edge of the obtained sheath area is the sheath edge. The sheath edge position is automatically obtained after solving model 1. Although automatic determination of the sheath edge position is a major benefit of the SASS model, the SASS model has a limitation as mentioned in the introduction.

In the presheath region, which is located between the sheath edge and the geometry edge, $V$ should be positive if the constraint given by Eq. (2) is not applied. In model 1, however, such a presheath region is forced to have the sheath-edge characteristics of $V=0, n_{\mathrm{e}}=n_{\mathrm{s}}$, and $u_{\mathrm{i}}=u_{\mathrm{B}}$ owing to the constraints given by Eqs. (2)-(4).

In a physically correct presheath model, the ion velocity should reach the Bohm velocity $u_{\mathrm{B}}$ at the sheath edge after traveling from the plasma-presheath interface to the sheath edge. In the case of model 1 mentioned above, the ion velocity already has the Bohm velocity in the presheath before reaching the sheath edge, which is physically unacceptable. Because of this, we must search for the sheath edges as the initial positions to calculate ion trajectories only in the physically correct sheath region. This search procedure is an easy task in the case of $1 \mathrm{D}$ geometries. However, it becomes a time-consuming and troublesome procedure in the case of $2 \mathrm{D}$ or $3 \mathrm{D}$ geometries, especially for those with complex structures.

\subsection{Boundary conditions for model 1}

The boundary conditions for model 1 are set as follows:

$$
\begin{array}{cll}
n_{\mathrm{i}}=n_{\mathrm{e}}=n_{\mathrm{s}}=n_{0} \mathrm{e}^{-1 / 2} & (x=L), \\
u_{\mathrm{i}}=u_{\mathrm{B}} & (x=L), \\
V=0 & (x=L), \\
V=V_{\mathrm{dc}} & (x=0),
\end{array}
$$

where $V_{\mathrm{dc}}(\leq 0)$ is the potential at the negatively biased surface. $L$ is the size of the calculation area. As can be understood from Eqs. (5)-(7), model 1 forces the calculated results to have 
the characteristics of the sheath edge at the outermost edge $x=L$.

Note that this setting is not to set the sheath edge position at $x=L$ before we solve model 1. The sheath position is involved in the obtained solution but we have to search for it. The sheath position is obtained as the first position where the ion velocity becomes the Bohm velocity when we evaluate the ion velocity from the surface $(x=0)$ toward the geometry edge $(x=L)$.

\subsection{Electron density and ion velocity in modified SASS model}

In the modified SASS model, the constraints given by Eqs. (2) and (3) are modified as follows to treat the presheath region:

$$
\begin{aligned}
& n_{\mathrm{e}}= \begin{cases}n_{0} \exp \left(\frac{q_{0} V}{k_{\mathrm{B}} T_{\mathrm{e}}}\right) & (V \leq 0), \\
n_{0} & (V>0),\end{cases} \\
& u_{\mathrm{i}}^{2}=-\frac{2 q_{0} V}{M_{\mathrm{i}}} .
\end{aligned}
$$

Since the outermost edge of the calculation area is assumed to be the edge of bulk plasma, the potential $V$ is measured from the bulk plasma edge, where the plasma density is $n_{0}$. Note that the actual potential $\phi$ measured from the ground level is expressed as $V(\leq 0)+\phi_{\mathrm{p}}$, where $\phi_{\mathrm{p}}$ is the bulk plasma potential measured from the ground level. If we need to draw the actual potential profile measured from the ground level, we can simply shift the calculated $V$ by $\phi_{\mathrm{p}}$. Since the actual potential $\phi$ cannot exceed the plasma potential $\phi_{\mathrm{p}}$, positive values of $V$ are forbidden during iterative calculation procedures. The initial velocity of ions at the geometry edge is zero because the electric field is assumed to be zero in the bulk plasma. Accordingly, the formula for the ion velocity $u_{\mathrm{i}}$, which is given by Eq. (3) in model 1, is modified to Eq. (10) in the modified SASS model.

Note that the ion velocity is calculated using the equation for freefall motion in both the conventional and modified SASS models. Thus, the ions in the entire geometry should be under a non- or weakly collisional condition. According to Meyer et al., ${ }^{53)}$ the presheath length is on the order of the mean free path $\lambda_{\text {inc }}$ of ions in the case of an electron cyclotron resonance etcher. This means that the modified SASS model can be applied to a practical processing plasma, although $L$ should be on the order of $\lambda_{\text {inc }}$. 


\subsection{Ion density in modified SASS model}

In the modified SASS model, the formula to obtain the ion density $n_{\mathrm{i}}$ is modified from Eq. (4) to the following formula:

$$
n_{\mathrm{i}}= \begin{cases}n_{\mathrm{s}} \frac{u_{\mathrm{B}}}{u_{\mathrm{i}}} & \left(u_{\mathrm{i}}^{2} \geq u_{\mathrm{B}}^{2}\right), \\ n_{\mathrm{e}}+\delta n & \left(u_{\mathrm{i}}^{2}<u_{\mathrm{B}}^{2}\right) .\end{cases}
$$

As can be understood from Eq. (11), we do not apply exact neutrality $\left(n_{\mathrm{i}}=n_{\mathrm{e}}\right)$ in the presheath but apply quasi-neutrality $\left(n_{\mathrm{i}}=n_{\mathrm{e}}+\delta n\right)$. $\delta n$ represents the deviation from exact neutrality, which is assumed to be small but not zero and can be estimated using the following equation if the transport of ions and electrons is governed by ambipolar diffusion: ${ }^{54}$ )

$$
\delta n \approx \frac{\varepsilon_{0}}{q_{0}} \frac{|\nabla V|}{L_{\mathrm{ch}}} \approx \frac{\varepsilon_{0}}{q_{0}} \frac{|V|}{L_{\mathrm{ch}}^{2}} .
$$

$\nabla V$ is the ambipolar electric field, which is very small in comparison with the electric field in the sheath but is not zero. $L_{\mathrm{ch}}$ is the characteristic length of the system. In this work, we assumed the following relationship:

$$
L_{\mathrm{ch}}=\sqrt{\frac{D_{\mathrm{a}}}{v_{\mathrm{izc}}}}
$$

where $D_{\mathrm{a}}$ is the ambipolar diffusion coefficient and $v_{\mathrm{izc}}$ is the ionization frequency in the bulk plasma. We assumed this relationship according to the fact that a time-independent diffusion equation is derived from Poisson's equation involving the space charge density $\delta n$ given by Eq. (12), which is described in the appendix. To investigate the effects of $\delta n$, we have examined the two modified SASS models of model 2 with $\delta n=0$ and model 3 with $\delta n$ given by Eq. (12).

\subsection{Boundary conditions for modified SASS model}

Since the outermost edge of the geometry is not the sheath edge but bulk plasma in the modified SASS model, the boundary conditions are modified as follows:

$$
\begin{array}{rlr}
n_{\mathrm{i}}=n_{\mathrm{e}} & =n_{0} & (x=L), \\
u_{\mathrm{i}} & =0 & (x=L), \\
V & =0 & (x=L), \\
V & =V_{\mathrm{dc}} & (x=0) .
\end{array}
$$

$L$ is the length from the biased surface to the edge of the bulk plasma and should be less than $\lambda_{\text {inc }}$ for ions according to the strict constraint on ion-neutral collisions. Generally, 
however, if the number of collisions is sufficiently small, we can apply noncollisional models even in the case of $L>\lambda_{\mathrm{i}}$. Such models are known as weakly collisional models. ${ }^{55)}$ Thus, we can apply the modified SASS models to $2 \mathrm{D}$ or $3 \mathrm{D}$ geometries in which the length of a diagonal can be $\sqrt{2}$ or $\sqrt{3}$ times larger than the length of a side.

Note that the negative bias $V_{\mathrm{dc}}$ is measured from the bulk plasma. Thus, $V_{\mathrm{dc}}$ is expressed as the sum of $\phi_{\mathrm{dc}}(\leq 0)$ and $\phi_{\mathrm{p}}$, where $\phi_{\mathrm{dc}}$ and $\phi_{\mathrm{p}}$ are the negative bias on the surface and the plasma potential measured from the ground level, respectively. If we need to perform the simulation on a nonbiased surface with $\phi_{\mathrm{dc}}=0$, we can set $V_{\mathrm{dc}}=-\phi_{\mathrm{p}}$.

\subsection{Calculation conditions}

We solved Poisson's equation using a commercially available finite element method solver, COMSOL Multiphysics, using the following parameters: plasma density $n_{0}=10^{17} \mathrm{~m}^{-3}$, electron temperature $T_{\mathrm{e}}=11,604 \mathrm{~K}(=1 \mathrm{eV})$, negative bias $V_{\mathrm{dc}}=-100 \mathrm{~V}$, ion mass $M_{\mathrm{i}}=$ $6.64 \times 10^{-26} \mathrm{~kg}$ (argon, atomic mass 40 ), pressure $P=1 \mathrm{mTorr}$, gas temperature $T_{\mathrm{g}}=300 \mathrm{~K}$, ion temperature $T_{\mathrm{i}}=400 \mathrm{~K}$. The Bohm velocity under these conditions is $u_{\mathrm{B}}=1,553 \mathrm{~m} / \mathrm{s}$. The Debye lengths in the bulk plasma and at the sheath edge are respectively as follows:

$$
\begin{aligned}
& \lambda_{\mathrm{De}}=\sqrt{\frac{\varepsilon_{0} k_{\mathrm{B}} T_{\mathrm{e}}}{n_{0} q_{0}^{2}}}=23.5 \mu \mathrm{m}, \\
& \lambda_{\mathrm{Ds}}=\sqrt{\frac{\varepsilon_{0} k_{\mathrm{B}} T_{\mathrm{e}}}{n_{\mathrm{s}} q_{0}^{2}}}=30.2 \mu \mathrm{m} .
\end{aligned}
$$

The thickness of the Child-Langmuir sheath is

$$
s_{\mathrm{CL}}=\lambda_{\mathrm{Ds}} \frac{\sqrt{2}}{3}\left(\frac{2\left|V_{\mathrm{dc}}\right|}{T_{\mathrm{e}}}\right)^{3 / 4}=0.757 \mathrm{~mm}=32 \lambda_{\mathrm{De}}
$$

Since the thickness of the Debye sheath $s_{\mathrm{De}}$ is approximately $5 \lambda_{\mathrm{Ds}}$ or $6 \lambda_{\mathrm{De}},{ }^{56)}$ the total thickness of the sheath is $s=s_{\mathrm{CL}}+s_{\mathrm{De}}=38 \lambda_{\mathrm{De}}$.

$\lambda_{\text {inc }}$ for ions, which is the maximum length for which we can apply modified SASS models, is ${ }^{57,58)}$

$$
\lambda_{\mathrm{i}}=\frac{1}{n_{\mathrm{g}} \sigma_{\mathrm{i}}}=41.4 \mathrm{~mm}=1,762 \lambda_{\text {De }},
$$

where $n_{\mathrm{g}}$ is the gas density, which is $6.44 \times 10^{19} \mathrm{~m}^{-3}$ under the present conditions. $\sigma_{\mathrm{i}}$ is the cross section of ion-neutral collisions, which is $10^{-18} \mathrm{~m}^{2}$ in the case of argon ions in argon plasma. ${ }^{57,59)}$ 

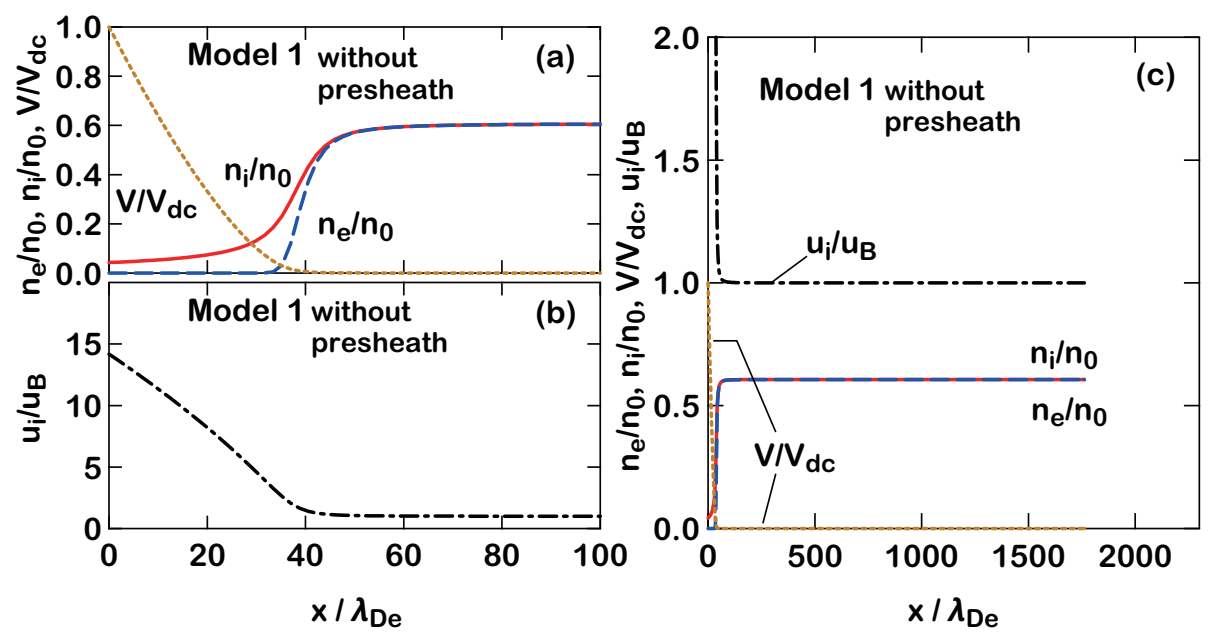

Fig. 1. (Color online) Calculated results of model 1 without a presheath region. Panels (a) and (b) show the results near the surface $\left(x / \lambda_{\text {De }}=0-100\right)$. Panel (c) shows those in the entire calculation region $\left(x / \lambda_{\mathrm{De}}=0-1750\right)$.

\section{Results and discussion}

\subsection{Model 1}

The results of the conventional SASS model (model 1) are shown in Figs. 1(a)-1(c). Figures 1(a) and 1(b) show the part of the results near the surface, in which we can see typical sheath characteristics at positions below the estimated sheath edge of $38 \lambda_{\mathrm{De}}$.

Figure 1(c), which covers the whole region, however, clearly shows problems with the conventional SASS model. At the left-hand side of the sheath edge, the electron and ion densities $n_{\mathrm{e}}$ and $n_{\mathrm{i}}$, respectively, are fixed at the sheath edge density $n_{\mathrm{s}}=0.6 n_{0}$. These characteristics are physically unacceptable because they should asymptotically approach the bulk plasma density $n_{0}$. In addition, the ion velocity $u_{\mathrm{i}}$ is fixed at the Bohm velocity in the region corresponding to the presheath. This characteristic is also physically unacceptable because $u_{\mathrm{i}}$ should gradually reach the Bohm velocity $u_{\mathrm{B}}$ as ions travel from the bulk plasma edge to the sheath edge. As can be understood from these results, model 1 generates physically unacceptable results in the region corresponding to the presheath. Therefore, physically correct ion trajectories are available only in the sheath region.

\subsection{Model 2}

In model 2, the constraints for solving Poisson's equation have been modified to handle the presheath region, but quasi-neutrality in the presheath is treated as exact neutrality. The results of model 2 are shown in Figs. 2(a)-2(c). 

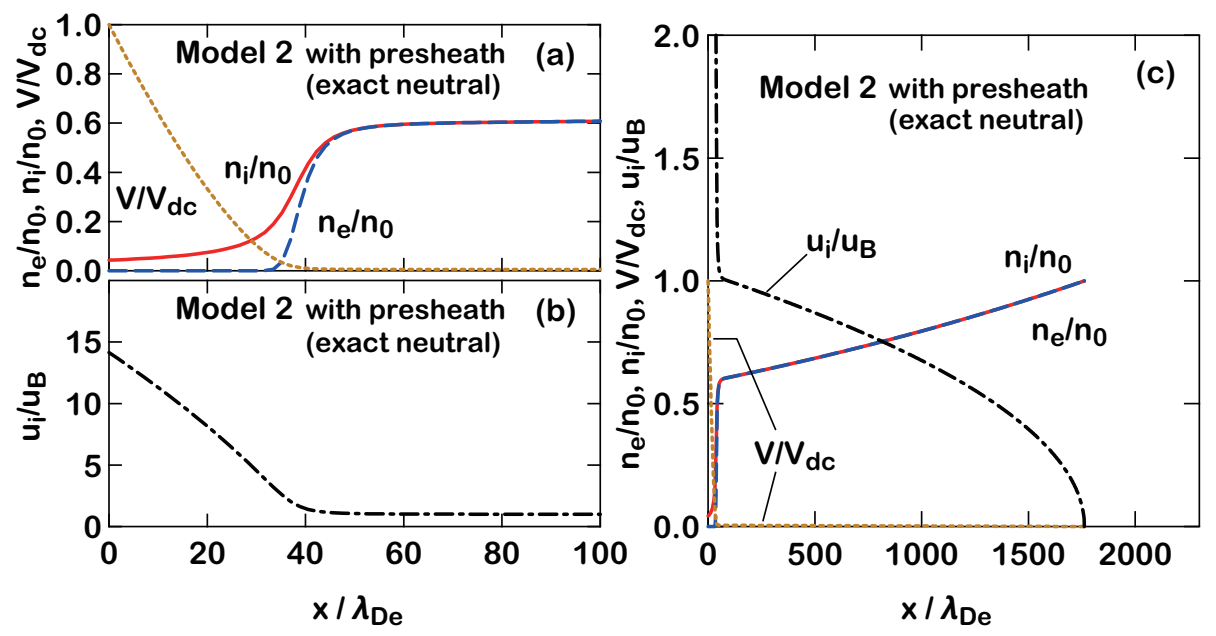

Fig. 2. (Color online) Calculated results of model 2 with an exact neutral presheath region. Panels (a) and (b) show the results near the surface $\left(x / \lambda_{\mathrm{De}}=0-100\right)$. Panel (c) shows those in the entire calculation region $\left(x / \lambda_{\mathrm{De}}=0-1750\right)$.

In model 2, as shown in Fig. 2(c), the electron and ion densities $n_{\mathrm{e}}$ and $n_{\mathrm{i}}$ gradually increase from the sheath edge toward the bulk plasma edge, while those in model 1 are fixed at the sheath edge plasma density $n_{\mathrm{s}}$. However, $n_{\mathrm{e}}$ and $n_{\mathrm{i}}$ in the presheath region do not asymptotically approach the plasma density $n_{0}$ but increase linearly to $n_{0}$. This characteristic is still physically unacceptable.

This is due to the fact that the quasi-neutrality in the presheath is treated as exact neutrality, which results in the potential $V$ having linear characteristics in the presheath. Since the potential in the presheath is sufficiently small to approximate the electron density as

$$
n_{\mathrm{e}}=n_{0} \exp \left(\frac{q_{0} V}{k_{\mathrm{B}} T_{\mathrm{e}}}\right) \approx n_{0}\left(1+\frac{q_{0} V}{k_{\mathrm{B}} T_{\mathrm{e}}}\right),
$$

the electron density $n_{\mathrm{e}}$ also has linear characteristics. At the same time, the ion density $n_{\mathrm{i}}$ also has the same characteristics because of the exact charge neutrality in the presheath.

The ion velocity $u_{\mathrm{i}}$ shows parabolic characteristics because $u_{\mathrm{i}}$ is proportional to $\sqrt{V}$, as can be understood from Eq. (10), and $V$ has linear characteristics as explained above. This feature of $V$ is the cause of the rapid increase in $u_{\mathrm{i}}$ when ions enter the presheath from the bulk plasma. This tendency of $u_{\mathrm{i}}$ disagrees with the physically acceptable picture of a presheath obtained from the exact model calculations, in which ions gradually accelerate from the bulk plasma edge toward the sheath edge..$^{29,30,43,60-62)}$

These results indicate that model 2 is not suitable for handling the presheath region. As can be understood from the above discussion, the main cause of this problem is the fact that the presheath region is treated as an exact neutral region. In the next subsection, we discuss 

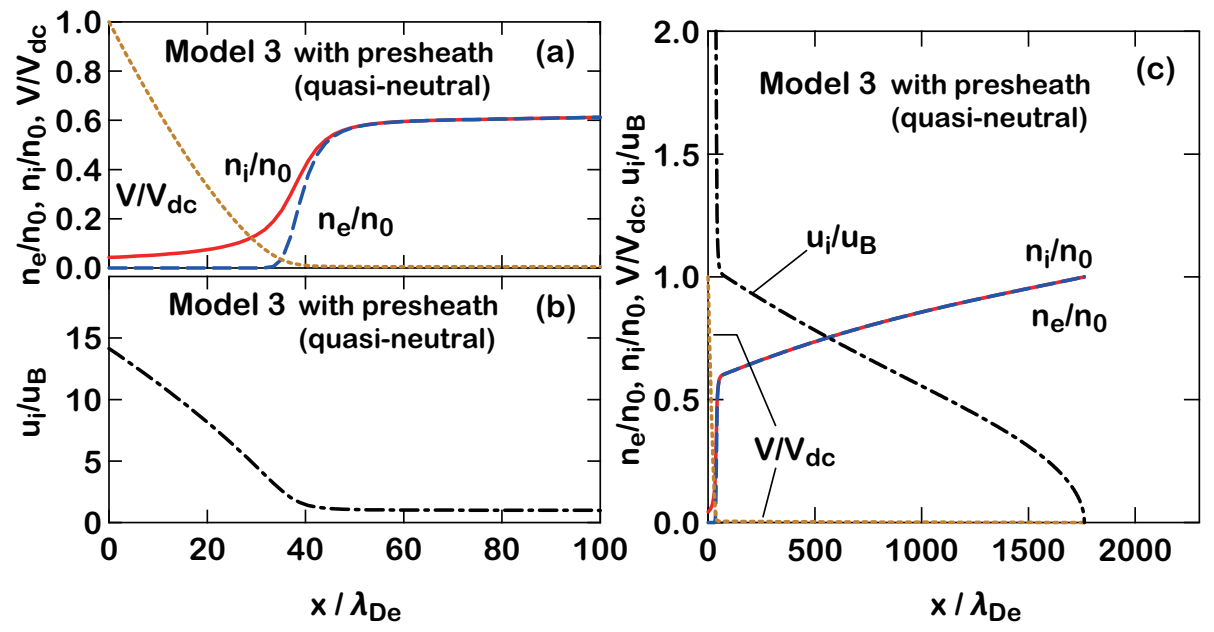

Fig. 3. (Color online) Calculated results of model 3 with a quasi-neutral presheath region. Panels (a) and (b) show the results near the surface $\left(x / \lambda_{\mathrm{De}}=0-100\right)$. Panel (c) shows those in the entire calculation region $\left(x / \lambda_{\text {De }}=0-1750\right)$.

improvements of the results in the presheath region by considering the slight non-neutrality involved in the quasi-neutral presheath region.

Note that the results in the sheath region obtained by using models 1 and 2 show exactly the same characteristics as can be understood from (a) and (b) of Figs. 1 and 2. This is due to the fact that these two models treat the sheath region in the same way.

\subsection{Model 3}

The presheath region, which connects the sheath and bulk plasma, is generally treated as a quasi-neutral region. In most cases, including model 2 in our study, the quasi-neutrality is treated in the same way as exact neutrality. ${ }^{36)}$ However, as discussed in the previous section, the application of exact neutrality to the presheath region results in physically unacceptable results. In this section, we discuss the results of model 3, which treats the quasi-neutral presheath as a region with space charge density $\delta n$ given by Eq. (12).

The results obtained by using model 3 are shown in Fig. 3. The electron and ion densities $n_{\mathrm{e}}$ and $n_{\mathrm{i}}$ do not show linear characteristics but slightly convex characteristics. As a result, the spatial distributions of $n_{\mathrm{e}}$ and $n_{\mathrm{i}}$ in the presheath region tend to asymptotically approach the bulk plasma density $n_{0}$. In addition, the spatial distribution of the ion velocity $u_{\mathrm{i}}$ becomes closer to the linear characteristic than the parabolic one.

Note again that the results in the sheath region obtained by using models 1, 2, and 3 show exactly the same characteristics as can be understood from (a) and (b) of Figs. 1, 2, and 3. 

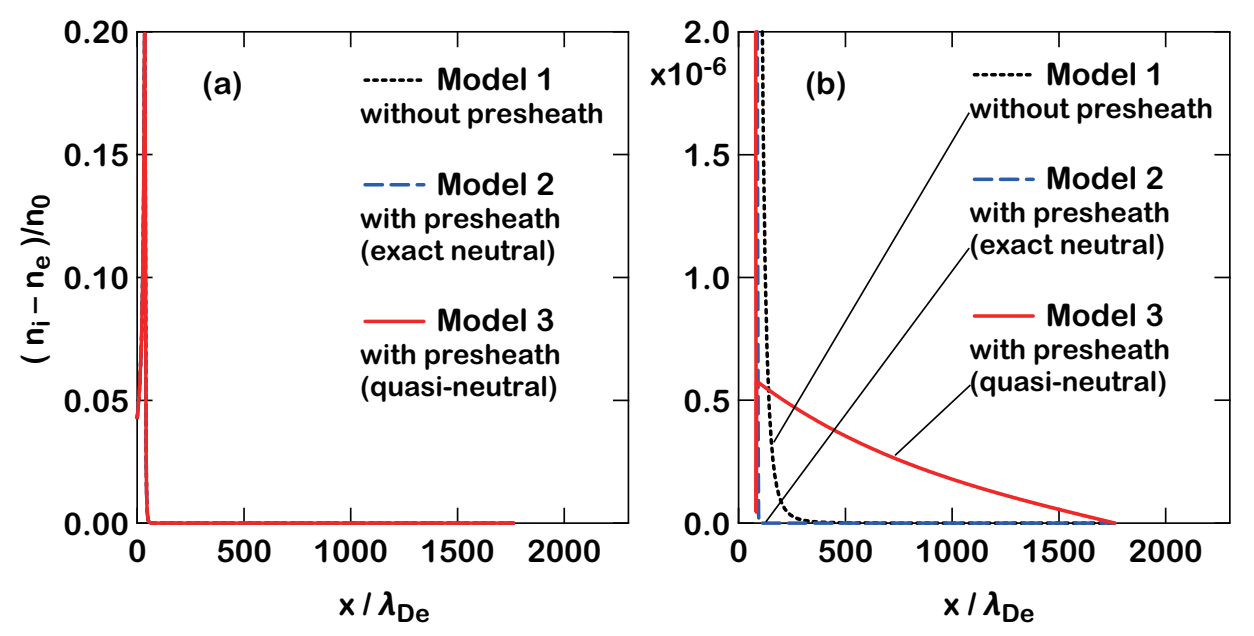

Fig. 4. (Color online) (a) Normalized space charge density profiles and (b) their magnified $\left(\times 10^{7}\right)$ profiles.

This is due to the fact that these three models treat the sheath region in the same way and means that the modifications of the model employed in this study do not alter the sheath region and only alter the presheath region.

\subsection{Space charge density, potential, and electric field in the presheath}

Figure 4(a) shows spatial profiles of the charge density obtained by using models 1, 2, and 3 . Figure 4(b) shows their $10^{7}$-fold magnified profiles. These three profiles seem to be identical according to the nonmagnified profiles shown in Fig. 4(a). On the other hand, looking at the magnified profiles shown in Fig. 4(b), we can see that only the result of model 3 has finite charge density, which is introduced as $\delta n$ given by Eq. (12), on the right-hand side of the sheath edge. This space charge density alters the spatial profiles of $V, n_{\mathrm{e}}, n_{\mathrm{i}}$, and $u_{\mathrm{i}}$ through Poisson's equation and the constraints, which is discussed later.

Figures 1-3 show the potential profiles obtained by using models 1, 2, and 3, respectively. Looking at the profiles at the scale in these figures, we can see that these potential profiles seem to be identical. However, we can see differences in the presheath region in the magnified profiles shown in Fig. 5(a). Figure 5(b) shows the corresponding electric field profiles, which are normalized by $E_{0}=\left(T_{\mathrm{e}} / 2\right) / \lambda_{\mathrm{i}}$.

The potential $V$ obtained by using model 1 becomes zero because of the constraints employed in model 1. In addition, the electric field also becomes zero, as shown in Fig. 5(b), for the same reason. On the other hand, in models 2 and 3, a finite electric field is formed in the presheath region.

In model 2, in which we apply exact neutrality in the presheath, the potential has a linear 

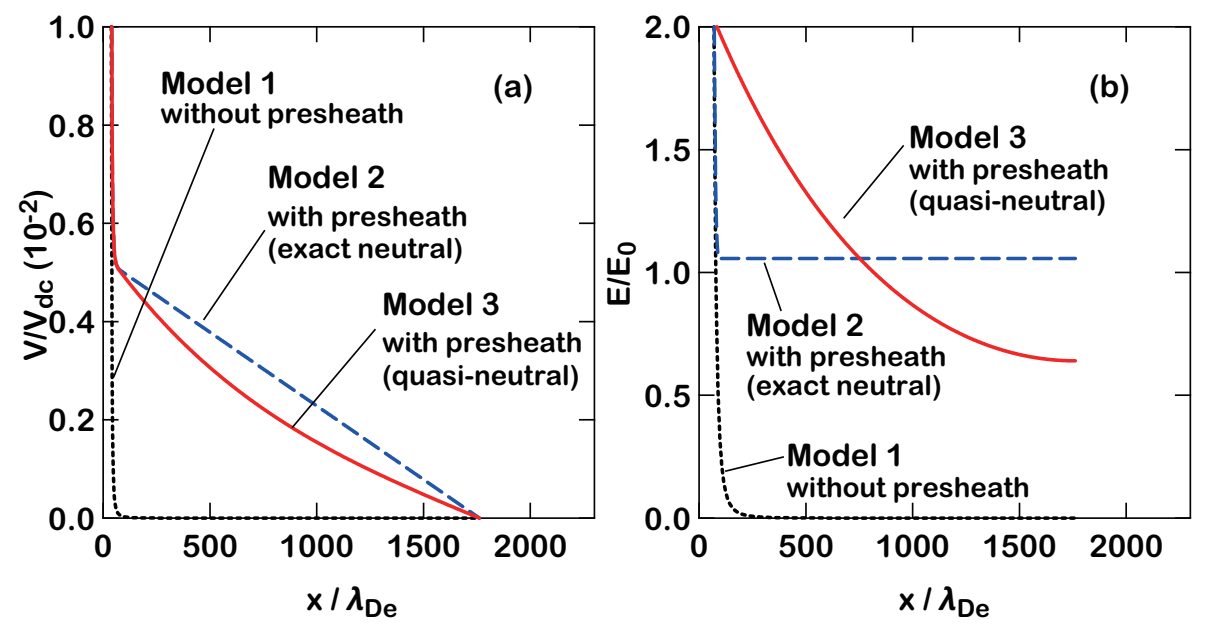

Fig. 5. (Color online) (a) Normalized potential profiles and (b) normalized electric field profiles.

profile with a slope of $E_{0}$, and the electric field becomes $E_{0}$. This can be easily understood by assuming the right-hand side of Poisson's equation to be zero. As described in the previous section, these characteristics cause linear increases in the electron and ion densities and the parabolic profile of the ion velocity as shown in Fig. 2(c).

In model 3, in which we apply slight non-neutrality $\delta n$ in the presheath, the potential, electric field, and $\delta n$ are self-consistently determined to satisfy Poisson's equation and the other constraints in model 3. As a result, the potential profile becomes concave, which is the main reason why model 3 shows presheath characteristics that are closer to those obtained by using the exact equations governing the presheath region.

\subsection{Comparison with experimental results}

We have checked the validity of our model through comparison with the experimental results given by Meyer et al., ${ }^{53)}$ in which presheath potential profiles were measured on a wafer stage in a downflow region of a $\mathrm{N}_{2}$ ECR plasma source. $D_{\mathrm{a}}$ was calculated to be $4 \mathrm{~m}^{2} / \mathrm{s}$ using the reported diffusion coefficients and mobilities of electrons obtained by BOLSIG+ ${ }^{63)}$ and those of ions reported by Moseley et al. ${ }^{64,65)} v_{\text {izc }}$ is not easy to determine but can be estimated from the types of plasma sources employed and the operation conditions. Since the report of Meyer et al. does not contain information of the ionization frequency, we employed $v_{\mathrm{izc}}=16.1 \mathrm{kHz}$ to obtain the best-fitting results. $L_{\mathrm{ch}}$ becomes $1.6 \mathrm{~cm}$ using these values of $D_{\mathrm{a}}$ and $v_{\mathrm{izc}}$.

Calculated potential profiles are shown in Fig. 6 together with the experimental results of Meyer et al. ${ }^{53)}$ Since their experimental potential profiles were measured from the ground level, we shifted our calculated potential $V$ by the actual plasma potential $\phi_{\mathrm{p}}$. The value 


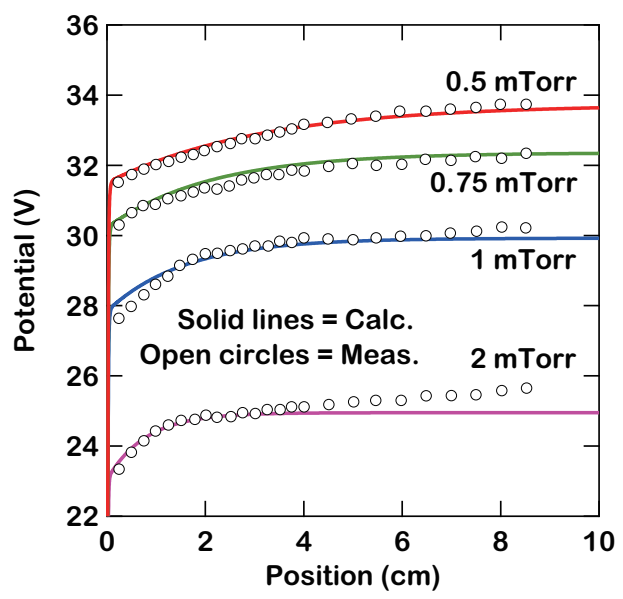

Fig. 6. (Color online) Comparison of experimentally obtained presheath profiles and numerical results obtained by using model 3.

of $\phi_{\mathrm{p}}$ for each pressure is regarded as the maximum value of the experimental potential. However, we slightly adjusted the value of $\phi_{\mathrm{p}}$ to obtain the best fit between the experimental and calculated potential profiles as shown in Fig. 6. We can see a fairly good agreement between the curvatures of the experimental and calculated potential profiles except for the case of 2 mTorr. The disagreement in the case of 2 mTorr may be due to deviation from the weakly collisional condition assumed in our model.

\subsection{Application to 2D geometries}

Since our model does not solve transport equations, the calculation time for obtaining the sheath and presheath characteristics becomes much shorter than that for conventional methods. In this subsection, we show examples of calculated results in 2D and 3D geometries.

Figure 7(a) shows calculated results of model 3 on a 2D geometry, in which a small convex object is placed on a surface biased to $-100 \mathrm{~V}$ on a plasma in $\mathrm{N}_{2}$ under a pressure of 1 mTorr. The electron temperature $T_{\mathrm{e}}$ and the plasma density $n_{0}$ are $3.8 \mathrm{eV}$ and $1.5 \times 10^{17}$ $\mathrm{m}^{-3}$, respectively. Figure 7(b) shows the calculated results of model 1 for comparison. Each figure shows spatial profiles of the potential normalized by $V_{\mathrm{dc}}$, its 100 -fold magnified view, and ion trajectories with the color indicating the ion velocity $u_{\mathrm{i}}$ normalized by $u_{\mathrm{B}}$.

Figure 8(a) is an enlarged view in the vicinity of the convex object at the bottom in Fig. 7(a). We can confirm that the sheath, namely, a steep potential drop, is formed near the convex object. Ion trajectories are bent by this potential profile. Note that it takes only 26 and $15 \mathrm{~s}$ to calculate the potential profile and ion trajectories, respectively, shown in Fig. 7(a) or 8(a), where the calculation was performed by using COMSOL and a Xeon E3-1240 processor (3.3 


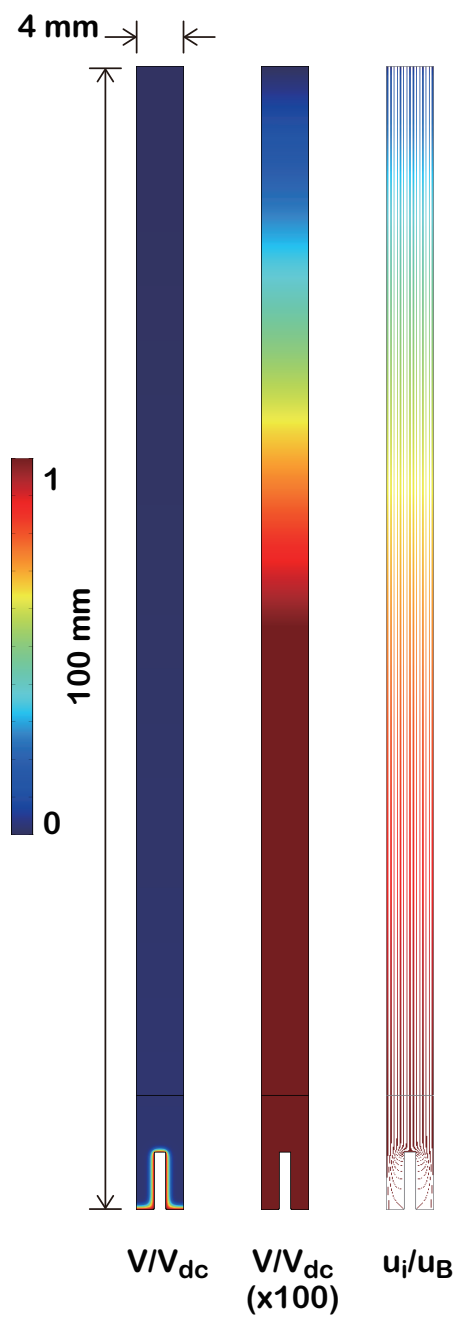

(a)

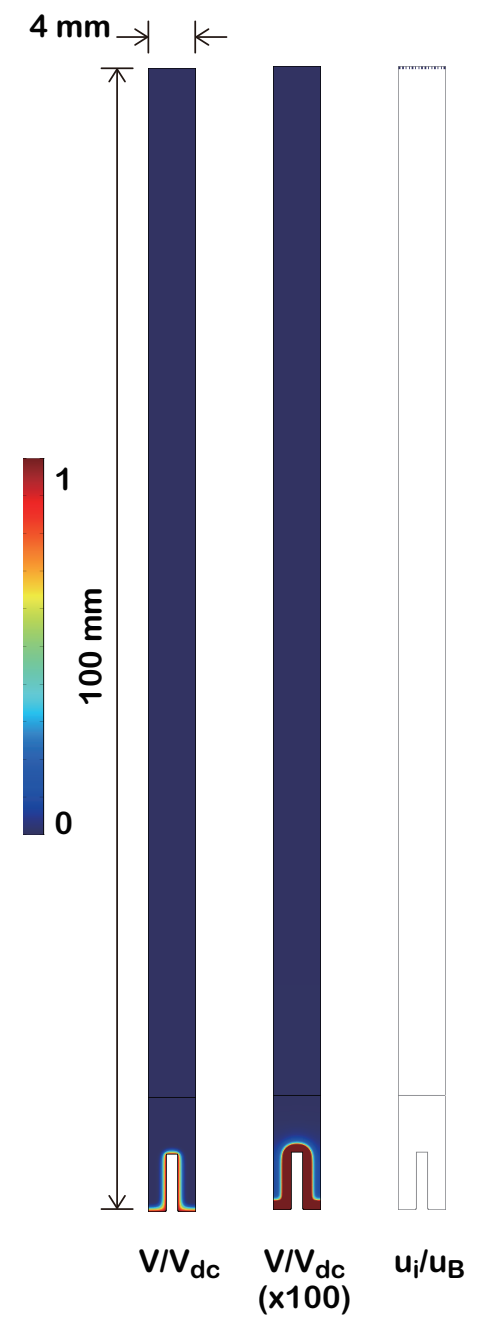

(b)

Fig. 7. (Color online) Calculated results of (a) model 3 (with presheath) and (b) model 1 (without presheath) applied to 2D geometry, in which a small convex object is placed on a negatively biased surface.

$\mathrm{GHz}$ ).

The potential profile obtained by using model 3, which was calculated in consideration of the presheath region, has a very small potential gradient in the presheath region in addition to the large potential drop near the convex object at the bottom. Although the small potential drop in the presheath cannot be confirmed in the figure plotted in the 1-fold scale of $V / V_{\mathrm{dc}}$, it can be clearly seen in the figure plotted in the 100 -fold scale of $V / V_{\mathrm{dc}}$, as shown in Fig. 7 (a). Ions in the presheath increase their velocities in this presheath region, which can be confirmed from the color change in the spatial profile of $u_{\mathrm{i}} / u_{\mathrm{B}}$.

The potential profiles obtained by using model 1, which cannot handle the presheath region, are almost the same as long as the figure is plotted in the 1-fold scale of $V / V_{\mathrm{dc}}$ as 



$u_{i} / u_{B}$

$u_{i} / u_{B}$

(a)

(b)

Fig. 8. (Color online) Magnified views in the vicinity of sheath regions in the calculated results of (a) model 3 (with presheath) and (b) model 1 (without presheath) applied to 2D geometry.

can be seen in Fig. 7(b). However, when we compare the potential profiles of models 1 and 3 in the 100 -fold scale of $V / V_{\mathrm{dc}}$, we can see clear differences between them. The potential drop in the presheath region in model 1 is exactly zero, while that in model 3 is not zero as mentioned before. Because of this feature of model 1, ions cannot increase velocity in the presheath region. Consequently, we cannot calculate the trajectories of ions, as can be seen in the spatial profile of $u_{\mathrm{i}} / u_{\mathrm{B}}$ in Fig. 7(b).

Figure 8(b) is an enlarged view of the vicinity of the convex object at the bottom in Fig. 7(b). We can confirm that the sheath formed in model 1 is almost exactly the same as that in model 3. However, since there is no potential drop in the presheath region in model 1, ions 


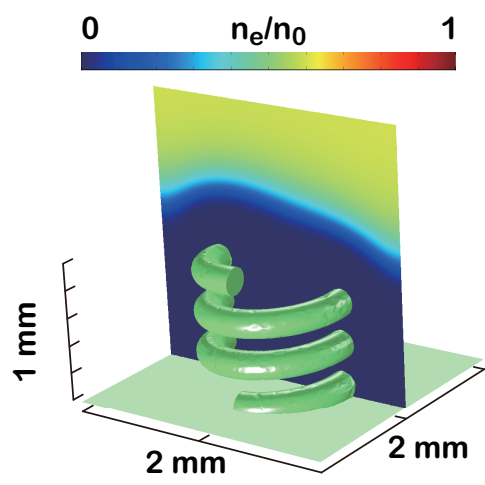

(a)

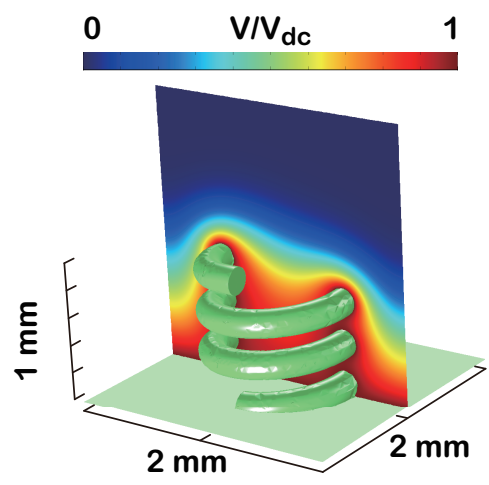

(c)

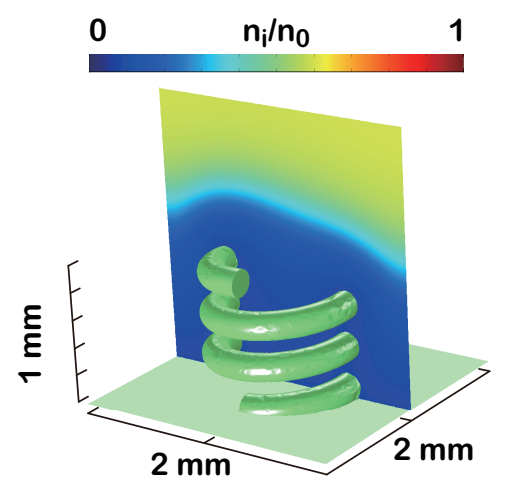

(b)



(d)

Fig. 9. (Color online) Application of model 3 to a 3D geometry containing a complex object on a negatively biased surface: (a) normalized electron density, (b) normalized ion density, (c) normalized potential, and (d) ion trajectories and their normalized velocities. Presheath regions are also calculated but are not included in these figures for simplicity.

cannot reach this region as shown in Fig. 8(b). This is one of the physically unacceptable features of model 1 .

\subsection{Application to 3D geometries}

In this subsection, we demonstrate that our model (model 3) can easily be extended to 3D geometries and show that the calculation can be accomplished in a very short time. Figures 9(a)-9(d) show the electron density, ion density, potential profile, and ion trajectories, respectively, around a coiled object biased to $-100 \mathrm{~V}$ in $1 \mathrm{mTorr} \mathrm{N}_{2}$ plasma with a plasma density of $1.5 \times 10^{17} \mathrm{~m}^{-3}$ and an electron temperature of $3.8 \mathrm{eV}$. In this calculation, it takes only 16 min 37 s to calculate the data shown in Figs. 9(a)-9(c) by using COMSOL and a Xeon E3-1240 processor (3.3 GHz). Calculation of the ion trajectories shown in Fig. 9(d) requires additional calculation of the motion of ions in the calculated potential. This requires 
an additional calculation time but only of $1 \mathrm{~min} 29 \mathrm{~s}$.

\section{Conclusions}

A SASS model can provide spatial profiles of space charge density, potential, and ion velocity in a sheath region by simply solving Poisson's equation together with several constraints deduced from Bohm's sheath theory. However, the conventional SASS model cannot handle a presheath region. We have modified the SASS model to be able to appropriately handle a presheath region by considering the small deviation from charge neutrality in the presheath.

\section{Acknowledgment}

This work was been partly supported by KAKENHI Grant Numbers 15H03585 and $15 \mathrm{~K} 13391$. 


\section{Appendix}

Supposing that $\delta n$ given by Eq. (12) is the space charge density in the presheath region, Poisson's equation Eq. (1) becomes the Helmholtz equation as follows:

$$
\nabla^{2} V-\frac{V}{L_{\mathrm{ch}}^{2}}=0 .
$$

The potential $V$ in the presheath region is generally very small, and the plasma density $n$ can be approximated as

$$
n=n_{0} \exp \left(\frac{q_{0} V}{k_{\mathrm{B}} T_{\mathrm{e}}}\right) \approx n_{0}\left(1+\frac{q_{0} V}{k_{\mathrm{B}} T_{\mathrm{e}}}\right) .
$$

Using Eq. (A·2), Eq. (A·1) for the potential $V$ is converted to the following equation for the plasma density $n$ :

$$
\nabla^{2} n-\frac{n}{L_{\mathrm{ch}}^{2}}=-\frac{n_{0}}{L_{\mathrm{ch}}^{2}}
$$

This equation is a time-independent diffusion equation with a nonzero source term corresponding to the right-hand side of this equation. This means that, in the presheath region with small $V$ and $\delta n$, solving Poisson's equation corresponds to solving the transport equation accompanied by the generation of electrons and ions through ionization.

The diffusion of charged particles in a presheath region is governed by ambipolar diffusion. In the diffusion equation for ambipolar diffusion, $L_{\mathrm{ch}}^{2}$ is given by $D_{\mathrm{a}} / v_{\mathrm{izc}}$, where $D_{\mathrm{a}}$ is the ambipolar diffusion coefficient and $v_{\mathrm{izc}}$ is the ionization frequency in the bulk plasma. ${ }^{66)} D_{\mathrm{a}}$ can be calculated using reported diffusion coefficients and mobilities of electrons and ions, ${ }^{67)}$ and is $576 \mathrm{~m}^{2} / \mathrm{s} . v_{\mathrm{izc}}$ is not easy to determine but can be estimated from the type of plasma source employed and the operation conditions. In this study, we employed $v_{\mathrm{izc}}=1.2 \mathrm{MHz}$ as a typical value for low-pressure argon plasma with a plasma density of $10^{17} \mathrm{~m}^{-3}{ }^{68)} L_{\mathrm{ch}}$ becomes $21.9 \mathrm{~mm}$ using these values of $D_{\mathrm{a}}$ and $v_{\mathrm{izc}}$. 


\section{References}

1) C. B. Zarowin and R. S. Horwath, J. Electrochem. Soc. 129, 2541 (1982).

2) J. W. Coburn and H. F. Winters, J. Vac. Sci. Technol. 16, 391 (1979).

3) J. W. Coburn and H. F. Winters, J. Appl. Phys. 50, 3189 (1979).

4) J.-K. Lee, S.-H. Lee, J.-H. Min, I.-Y. Jang, C.-K. Kim, and S. H. Moon, J. Electrochem. Soc. 156, D222 (2009).

5) S. A. Cybart, P. Roediger, E. Ulin-Avila, S. M. Wu, T. J. Wong, and R. C. Dynes, J. Vac. Sci. Technol. B 31, 010604 (2013).

6) D. B. Burckel, P. S. Finnegan, M. D. Henry, P. J. Resnick, and R. L. Jarecki, Jr., Appl. Phys. Lett. 108, 142103 (2016).

7) J. M. E. Harper, J. J. Cuomo, P. A. Leary, G. M. Summa, H. R. Kaufman, and F. J. Bresnock, J. Electrochem. Soc. 128, 1077 (1981).

8) D. L. Flamm and G. K. Herb, in Plasma Etching: An Introduction, ed. D. M. Manos and D. L. Flamm (Academic Press, San Diego, CA, 1989) Chap. 1.

9) G. S. Oehrlein and Y. H. Lee, J. Vac. Sci. Technol. A 5, 1585 (1987).

10) T. Ohchi, S. Kobayashi, M. Fukasawa, K. Kugimiya, T. Kinoshita, T. Takizawa, S. Hamaguchi, Y. Kamide, and T. Tatsumi, Jpn. J. Appl. Phys. 47, 5324 (2008).

11) T. Ito, K. Karahashi, K. Mizotani, M. Isobe, S.-Y. Kang, M. Honda, and S. Hamaguchi, Jpn. J. Appl. Phys. 51, 08 HB01 (2012).

12) K. Eriguchi, A. Matsuda, Y. Takao, and K. Ono, Jpn. J. Appl. Phys. 53, 03 DE02 (2014).

13) R. J. Hoekstra, M. J. Kushner, V. Sukharev, and P. Schoenborn, J. Vac. Sci. Technol. B 16, 2102 (1998).

14) G. Franz, J. Electrochem. Soc. 140, 1147 (1993).

15) N. Y. Babaeva and M. J. Kushner, J. Appl. Phys. 101, 113307 (2007).

16) N. Y. Babaeva and M. J. Kushner, J. Phys. D 41, 062004 (2008).

17) S. Takahashi, K. Suzuki, M. Okano, M. Imada, T. Nakamori, Y. Ota, K. Ishizaki, and S. Noda, Nat. Mater. 8, 721 (2009).

18) S. Takahashi, M. Okano, M. Imada, and S. Noda, Appl. Phys. Lett. 89, 123106 (2006).

19) E. Kawamura, M. A. Lieberman, and D. B. Graves, Plasma Sources Sci. Technol. 23, 064003 (2014).

20) L. Tonks and I. Langmuir, Phys. Rev. 34, 876 (1929).

21) D. Bohm, in The Characteristics of Electrical Discharges in Magnetic Field, ed. A. Guthrie and R. K. Wakerling (McGraw-Hill, New York, 1949) Chap. 3. 
22) R. N. Franklin and J. R. Ockendon, J. Plasma Phys. 4, 371 (1970).

23) V. A. Godyak, Phys. Lett. A 89, 80 (1982).

24) V. A. Godyak and N. Sternberg, IEEE Trans. Plasma Sci. 18, 159 (1990).

25) K.-U. Riemann, J. Phys. D 24, 493 (1991).

26) A. Kono, J. Phys. D 32, 1357 (1999).

27) R. N. Franklin and J. Snell, Phys. Plasmas 7, 3077 (2000).

28) R. N. Franklin, IEEE Trans. Plasma Sci. 30, 352 (2002).

29) V. Godyak and N. Sternberg, Phys. Plasmas 9, 4427 (2002).

30) R. N. Franklin, J. Phys. D 36, R309 (2003).

31) V. Godyak and N. Sternberg, IEEE Trans. Plasma Sci. 31, 303 (2003).

32) A. Kono, J. Phys. D 36, 465 (2003).

33) N. Hershkowitz, Phys. Plasmas 12, 055502 (2005).

34) K. U. Riemann, Phys. Plasmas 13, 063508 (2006).

35) N. Sternberg and V. Godyak, IEEE Trans. Plasma Sci. 35, 1341 (2007).

36) K.-U. Riemann, Plasma Sources Sci. Technol. 18, 014006 (2009).

37) M. Keidar and I. I. Beilis, IEEE Trans. Plasma Sci. 33, 1481 (2005).

38) Z. Sternovsky and S. Robertson, IEEE Trans. Plasma Sci. 34, 850 (2006).

39) J. E. Allen, Plasma Sources Sci. Technol. 18, 014004 (2009).

40) S. D. Baalrud and C. C. Hegna, Plasma Sources Sci. Technol. 20, 025013 (2011).

41) R. P. Brinkmann, J. Phys. D 44, 042002 (2011).

42) U. Czarnetzki, Phys. Rev. E 88, 063101 (2013).

43) S. Robertson, Plasma Phys. Control. Fusion 55, 093001 (2013).

44) B. Scheiner, S. D. Baalrud, B. T. Yee, M. M. Hopkins, and E. V. Barnat, Phys. Plasmas 22, 123520 (2015).

45) D. D. Tskhakaya, Sr., L. Kos, and D. Tskhakaya, Phys. Plasmas 23, 032128 (2016).

46) P. Cagas, A. Hakim, J. Juno, and B. Srinivasan, Phys. Plasmas 24, 022118 (2017).

47) E. Stamate, H. Sugai, O. Takai, and K. Ohe, J. Appl. Phys. 95, 830 (2004).

48) E. Stamate, N. Holtzer, and H. Sugai, Appl. Phys. Lett. 86, 261501 (2005).

49) E. Stamate and H. Sugai, Phys. Rev. E 72, 036407 (2005).

50) K. Denpoh and T. Shirafuji, Jpn. J. Appl. Phys. 48, 090209 (2009).

51) K. Denpoh and T. Shirafuji, Jpn. J. Appl. Phys. 49, 056202 (2010).

52) K. Denpoh and T. Shirafuji, Jpn. J. Appl. Phys. 50, 036001 (2011).

53) J. A. Meyer, G. H. Kim, M. J. Goeckner, and N. Hershkowitz, Plasma Sources Sci. Technol. 1, 147 (1992). 
54) B. M. Smirnov, Theory of Gas Discharge Plasma (Springer, Cham, 2015) Chap. 3.

55) E. Zawaideh, N. S. Kim, and F. Najmabadi, Phys. Fluids B 2, 647 (1990).

56) F. F. Chen and J. P. Chang, Lecture Notes on Principles of Plasma Processing (Springer, New York, 2002) Chap. 2.

57) M. A. Lieberman and A. J. Lichtenberg, Principles of Plasma Discharges and Materials Processing (Wiley, Hoboken, NJ, 2005) 2nd ed., p. 80.

58) E. W. McDaniel, Collision Phenomena in Ionized Gases (Wiley, New York, 1964) Chap. 2.

59) E. W. McDaniel, Collision Phenomena in Ionized Gases (Wiley, New York, 1964) p. 164.

60) S. D. Baalrud, B. Scheiner, B. Yee, M. Hopkins, and E. Barnat, Plasma Phys. Control. Fusion 57, 044003 (2015).

61) D. D. Tskhakaya, Sr., L. Kos, and N. Jelić, Phys. Plasmas 21, 073503 (2014).

62) A. Meige, G. Leray, J. L. Raimbault, and P. Chabert, Appl. Phys. Lett. 92, 061501 (2008).

63) G. J. M. Hagelaar and L. C. Pitchford, Plasma Sources Sci. Technol. 14, 722 (2005).

64) J. T. Moseley, R. M. Snuggs, D. W. Martin, and E. W. McDaniel, Phys. Rev. 178, 240 (1969).

65) J. T. Moseley, R. M. Snuggs, D. W. Martin, and E. W. McDaniel, Phys. Rev. Lett. 21, 873 (1968).

66) M. A. Lieberman and A. J. Lichtenberg, Principles of Plasma Discharges and Materials Processing (Wiley, Hoboken, NJ, 2005) 2nd ed., Chap. 5.

67) E. Gogolides and H. H. Sawin, J. Appl. Phys. 72, 3971 (1992).

68) P. Chabert and N. Braithwaite, Physics of Radio-Frequency Plasmas (Cambridge University Press, Cambridge, U.K., 2011) p. 33. 